\title{
Reflection and Ducting of Gravity Waves Inside the Sun
}

\author{
K.B. MacGregor · T.M. Rogers
}

Received: 7 January 2011 / Accepted: 6 April 2011 / Published online: 13 May 2011

(C) Springer Science+Business Media B.V. 2011

\begin{abstract}
Internal gravity waves excited by overshoot at the bottom of the convection zone can be influenced by rotation and by the strong toroidal magnetic field that is likely to be present in the solar tachocline. Using a simple Cartesian model, we show how waves with a vertical component of propagation can be reflected when traveling through a layer containing a horizontal magnetic field with a strength that varies with depth. This interaction can prevent a portion of the downward traveling wave energy flux from reaching the deep solar interior. If a highly reflecting magnetized layer is located some distance below the convection zone base, a duct or wave guide can be set up, wherein vertical propagation is restricted by successive reflections at the upper and lower boundaries. The presence of both upward and downward traveling disturbances inside the duct leads to the existence of a set of horizontally propagating modes that have significantly enhanced amplitudes. We point out that the helical structure of these waves makes them capable of generating an $\alpha$-effect, and briefly consider the possibility that propagation in a shear of sufficient strength could lead to instability, the result of wave growth due to over-reflection.
\end{abstract}

Keywords Solar interior · Solar magnetic field · Magnetohydrodynamics · Internal gravity waves

\section{Introduction}

The ways in which internal gravity waves can affect the compositional and dynamical states of the solar radiative interior have received considerable attention in recent years. For example, wave-induced mixing of the layers just below the convection zone has been invoked

K.B. MacGregor ( $\varangle)$

High Altitude Observatory, National Center for Atmospheric Research, 3080 Center Green, Boulder, CO 80301, USA

e-mail: kmac@ucar.edu

T.M. Rogers

Department of Planetary Sciences, University of Arizona, Tucson, AZ 85721, USA

e-mail: tamirogers@mac.com 
to account for the observed depletion of lithium in solar/stellar photospheres (Garcia-Lopez and Spruit, 1991; Schatzman, 1996; Fritts, Vadas, and Andreassen, 1998), and it has been suggested that internal waves can contribute to transport processes deep within the cores of the Sun and stars (Press, 1981; Press and Rybicki, 1981). The interaction between radiatively damped gravity waves and a mean shear flow, the forcing mechanism thought to be responsible for the observed quasi-biennial oscillation in the equatorial stratospheric layers of the Earth's atmosphere (see, e.g., Baldwin et al., 2001, and references therein), has been investigated in the context of the time-dependent dynamics of the tachocline and the underlying stable region (Kumar, Talon, and Zahn, 1999; Kim and MacGregor, 2001; Rogers, MacGregor, and Glatzmaier, 2008). There has also been much discussion concerning the effects of inwardly propagating waves on the overall internal solar rotation, particularly the long-term consequences of any angular-momentum redistribution caused by waves for the rotational state of the radiative zone of the Sun (see Ringot, 1998 and references therein for a summary). In this regard, it has been suggested (Talon, Kumar, and Zahn, 2002; Charbonnel and Talon, 2005; Talon and Charbonnel, 2005; see also Denissenkov, Pinsonneault, and MacGregor, 2008) that gravity-wave interactions in the tachocline region and in the deeper interior can act in concert to produce near-uniform rotation of the core, in accord with helioseismic inferences (see, e.g., Charbonneau et al., 1998).

In a stably and continuously stratified fluid in which the effects of compressibility can be neglected, hydrodynamic gravity waves are generated when a localized region is perturbed by a small-amplitude disturbance that varies over a time scale that is longer than the period of adiabatic buoyancy oscillations at that position. On this basis, the inner and outer bounding surfaces of the Sun's convective envelope are likely sites of internal-wave emission into the contiguous, stable layers of the solar interior and atmosphere. These interfaces are deformed by convective fluid motions that overshoot and penetrate adjacent stable regions where the local buoyancy period is typically short in comparison to the time scales characteristic of the perturbing flows. Kiraga et al. (2003), Rogers and Glatzmaier (2005a, 2005b) and Rogers, Glatzmaier, and Jones (2006) used detailed numerical simulations to study the excitation of gravity waves by overshooting plumes at the bottom of the Sun's convective envelope $\left(r \approx 0.71 R_{\odot}\right.$ ). The inward propagation of the waves generated in this way must take them through the tachocline, which helioseismic analyses indicate has a central radius and thickness at equatorial latitudes of about $0.69 R_{\odot}$ and $0.04 R_{\odot}$, respectively (Charbonneau et al., 1999). Numerous studies of flux-tube formation and dynamics have delineated the links between the bipolar magnetic regions that are observed to emerge at the solar surface and magnetic flux stored in the subadiabatic layers beneath the convection zone (see, e.g., Schüssler, 1996; Fisher et al., 2000). The results of these investigations point toward the existence of a strong $(\approx 10-100 \mathrm{kG})$, toroidal magnetic field within the tachocline, implying that the internal waves that traverse this region must be hydromagnetic rather than hydrodynamic in nature.

This article examines one potential consequence of magnetohydrodynamic (MHD) modifications to the properties of gravity waves inside the Sun: the possibility of reflection when a wave having a vertical component of propagation encounters magnetic conditions that change rapidly with depth below the convection zone. Some of the effects of a horizontal magnetic field at the bottom of the convection zone on the propagation of internal gravity waves into the radiative interior were investigated by Schatzman (1993). Since the length scale for radiative damping varies with wave frequency $[\omega]$ as $\omega^{4}$ (e.g., Kim and MacGregor, 2001), the effects of reflection are likely to be most relevant for higher-frequency waves that suffer less attenuation and are thus capable, in principle, of propagating into the deep interior of the Sun (Press, 1981). Not only might a highly reflecting magnetic layer prevent 
some portion of the downward traveling energy flux from reaching the inner solar core, but it could contribute to the trapping of vertically propagating waves within a horizontal layer directly beneath the convection zone. That is, successive reflections of a wave from the base of the adiabatically stratified convection zone (wherein gravity waves are evanescent) and an underlying, depth-dependent toroidal magnetic field can lead to the formation of a duct or wave guide, effectively confining the wave to the region between the upper and lower reflecting surfaces, and allowing for horizontal propagation only. The existence and properties of such ducts for internal-gravity waves has been studied by atmospheric physicists in connection with the development and propagation of squall lines (Lindzen and Tung, 1976).

In this article, we develop preliminary descriptions of gravity-wave reflection and ducting inside the Sun, using a simplified model for the magnetic and dynamical structure of the radiative layers comprised by the tachocline region. This article is a companion to the recent articles by Rogers and MacGregor (2010, 2011), providing a conceptual basis to interpret some of the detailed numerical results reported on in those articles. In Section 2, we derive the properties and propagation characteristics of MHD internal waves in a rotating, gravitationally stratified fluid. The results of this section are then used in Section 3 to derive continuity conditions that enable us to treat the reflection of obliquely propagating gravity waves from a discontinuity in the strength of an otherwise uniform, horizontal magnetic field. Among other things, we derive an expression for the wave-reflection coefficient and use it to deduce the combinations of wave frequencies and horizontal wavenumbers for which the reflecting layer can function as the bottom of a duct. We obtain a solution for forced, hydromagnetic gravity waves in a duct of specified vertical extent, and examine the properties of the modes supported by such a structure when its lower bounding surface is perfectly reflecting. In Section 4, we summarize our results and briefly consider a few implications of gravity-wave reflection and ducting for the MHD physics of the solar interior, including the helical structure of the waves and the possible relevance of this property to dynamo processes in the tachocline region, and the potential for over-reflection and instability in regions of strong shear.

\section{Gravity Waves in a Rotating, Magnetized Fluid}

When gravity is the only external force acting on an incompressible, stably stratified fluid, propagating disturbances take the form of internal waves, driven by the fluctuating pressure gradient and buoyancy forces (see, e.g., Turner, 1973; Lighthill, 1978). In the presence of rotation and a magnetic field, gravity-wave properties are modified through the additional influences of the Coriolis and Lorentz forces. To ascertain the nature of these changes, we consider infinitesimal, additive perturbations $\delta \rho, \delta p, \delta \mathbf{u}$, and $\delta \mathbf{B}$ to the density $\rho_{0}$, pressure $p_{0}$, velocity $\mathbf{u}_{0}$, and magnetic field $\mathbf{B}_{0}$ of the mean equilibrium state. For an ideal MHD fluid that rotates uniformly with a stationary angular velocity $\boldsymbol{\Omega}$, the behavior of these quantities is governed by the linearized equations

$$
\begin{gathered}
\nabla \cdot \delta \mathbf{u}=0, \\
\frac{\partial \delta \rho}{\partial t}+\delta \mathbf{u} \cdot \nabla \rho_{0}=0, \\
\rho_{0} \frac{\partial \delta \mathbf{u}}{\partial t}+2 \rho_{0} \mathbf{\Omega} \times \delta \mathbf{u}=-\nabla \delta p-\mathbf{g} \delta \rho+\frac{1}{4 \pi}\left[\left(\nabla \times \mathbf{B}_{0}\right) \times \delta \mathbf{B}+(\nabla \times \delta \mathbf{B}) \times \mathbf{B}_{0}\right],
\end{gathered}
$$




$$
\frac{\partial \delta \mathbf{B}}{\partial t}=\nabla \times\left(\delta \mathbf{u} \times \mathbf{B}_{0}\right),
$$

where $\mathbf{g}$ is the gravitational acceleration. The continuity equation (1) expresses the assumed incompressibility of the fluid; Equation (2) indicates that fluctuations in the density at any location result from the displacement of the mean density stratification by the fluctuating velocity field of the wave (Turner, 1973; Lighthill, 1978). Equations (3) and (4) are, respectively, the linearized versions of the momentum equation (in the rotating frame of reference) and the induction equation for the inviscid, perfectly electrically conducting fluid. For simplicity, we have assumed $\mathbf{u}_{0}=0$, with the unperturbed equilibrium state determined by the balance between the pressure gradient, gravitational, and magnetic forces; the effect of a background flow field will be discussed in Section 4.

We are ultimately interested in how the magnetic structure in the environs of the tachocline affects waves that propagate inward from the base of the convection zone. Since the radial extent of this region is estimated to be only $\approx 10^{-2} R_{\odot}$, we can facilitate the subsequent analysis by adopting a local Cartesian coordinate system with $x-, y$-, and $z-$ axes oriented parallel, respectively, to the directions of increasing $\theta, \phi$, and $r$ in a spherical coordinate system with origin at the center of the Sun and polar axis aligned with the solar rotation axis. Relative to this Cartesian system, $\mathbf{g}=-g \mathbf{e}_{z}$ with $g$ assumed constant, $\mathbf{B}_{0}=B_{0}(z) \mathbf{e}_{y}$, and $\boldsymbol{\Omega}=\Omega_{x} \mathbf{e}_{x}+\Omega_{z} \mathbf{e}_{z}$ with $\Omega_{x}=-\Omega \sin \theta$ and $\Omega_{z}=\Omega \cos \theta$. The first two of these assumptions imply that density $\left[\rho_{0}\right]$ and pressure $\left[p_{0}\right]$ of the equilibrium state in the absence of waves are functions of $z$ only.

The treatment is further simplified by using two additional approximations: $i$ ) the Boussinesq approximation (Spiegel and Veronis, 1960; Turner, 1973), whereby the variation in density is neglected in those terms in the momentum equation (3) involving fluid inertia but retained in the buoyant force; and ii) the $f$-plane approximation (see, e.g., Gill, 1982), under which the Coriolis force components arising from $\Omega_{x}$ in Equation (3) are dropped, but those due to $\Omega_{z}$ are kept. The first of these approximations requires that the vertical scale of the wave motion be smaller than the scale over which $\rho_{0}$ varies, a condition that is well satisfied in the radiative layers underlying the convection zone. The second approximation provides a reasonable description of wave dynamics for slowly rotating systems (i.e., when the rotation period is much longer than the period of adiabatic buoyancy oscillations), and when the domain of interest spans a narrow range in $\theta$ that is not located too near to the Equator. Although we confine our study to disturbances that fulfill these constraints, it is also straightforward to derive the wave properties presented herein without recourse to the $f$-plane approximation.

Equations (1) - (4) admit traveling-wave solutions of the general form $\delta Q=\delta \hat{Q}(z) \times$ $\exp [\mathrm{i}(k x+l y-\omega t)]$, where $\delta Q$ is any of the eight perturbation quantities $\delta \rho, \delta p, \delta \mathbf{u}$, and $\delta \mathbf{B}$. Through a process of repeated substitution and elimination, each of these perturbations can be expressed in terms of the vertical velocity fluctuation $\left[\delta u_{z}\right]$. An equation for the amplitude $\left[\delta \hat{u}_{z}(z)\right]$ can then be obtained by substituting the resulting expressions for the perturbations into the $z$-component of the momentum equation (3), yielding

$$
\frac{\mathrm{d}}{\mathrm{d} z}\left\{\left[\frac{\left(\omega^{2}-l^{2} u_{\mathrm{A}}^{2}\right)^{2}-\omega^{2} f^{2}}{\left(k^{2}+l^{2}\right)\left(\omega^{2}-l^{2} u_{\mathrm{A}}^{2}\right)}\right] \frac{\mathrm{d} \delta \hat{u}_{z}}{\mathrm{~d} z}\right\}+\left[N^{2}-\left(\omega^{2}-l^{2} u_{\mathrm{A}}^{2}\right)\right] \delta \hat{u}_{z}=0,
$$

where $u_{\mathrm{A}}=B_{0} / \sqrt{4 \pi \rho_{0}}$ is the Alfvén speed, $f=2 \Omega_{z}$ is the Coriolis parameter, and

$$
N=\left[-\left(g / \rho_{0}\right)\left(\mathrm{d} \rho_{0} / \mathrm{d} z\right)\right]^{1 / 2}
$$

is the Brunt-Väisälä or buoyancy frequency. 
As noted above, the remaining components of Equations (1)-(4) provide relations between $\delta \hat{u}_{z}$ and the other perturbation quantities. Specifically, the density fluctuation associated with the wave is given by

$$
\delta \hat{\rho}=\rho_{0}\left(\mathrm{i} N^{2} / g \omega\right) \delta \hat{u}_{z},
$$

while the $x$ - and $y$-components of the velocity amplitude are

$$
\delta \hat{u}_{x}=\left[\frac{\mathrm{i} k\left(\omega^{2}-l^{2} u_{\mathrm{A}}^{2}\right)-\omega f l}{\left(k^{2}+l^{2}\right)\left(\omega^{2}-l^{2} u_{\mathrm{A}}^{2}\right)}\right] \frac{\mathrm{d} \delta \hat{u}_{z}}{\mathrm{~d} z}
$$

and

$$
\delta \hat{u}_{y}=\left[\frac{\mathrm{i} l\left(\omega^{2}-l^{2} u_{\mathrm{A}}^{2}\right)+\omega f k}{\left(k^{2}+l^{2}\right)\left(\omega^{2}-l^{2} u_{\mathrm{A}}^{2}\right)}\right] \frac{\mathrm{d} \delta \hat{u}_{z}}{\mathrm{~d} z} .
$$

The induction equation (4) furnishes the means for determining $\delta \hat{\mathbf{B}}$ from $\delta \hat{\mathbf{u}}$,

$$
\begin{aligned}
& \delta \hat{B}_{x}=-B_{0} \frac{l \delta \hat{u}_{x}}{\omega} \\
& \delta \hat{B}_{y}=B_{0} \frac{k \delta \hat{u}_{x}}{\omega}-\frac{\mathrm{i}}{\omega} \frac{\mathrm{d}\left(B_{0} \delta \hat{u}_{z}\right)}{\mathrm{d} z}, \\
& \delta \hat{B}_{z}=-B_{0} \frac{l \delta \hat{u}_{z}}{\omega}
\end{aligned}
$$

with $\delta \hat{u}_{x}$ related to $\delta \hat{u}_{z}$ through Equation (8). Similarly, the total pressure perturbation [ $\left.\delta \hat{p}_{\text {tot }}\right]$ is the sum of the fluid and magnetic pressure perturbations, and is expressible in terms of $\delta \hat{u}_{z}$ as

$$
\delta \hat{p}_{\mathrm{tot}}=\delta \hat{p}+\frac{B_{0} \delta \hat{B}_{y}}{4 \pi}=\mathrm{i} \frac{\rho_{0}}{\omega}\left[\frac{\left(\omega^{2}-l^{2} u_{\mathrm{A}}^{2}\right)^{2}-\omega^{2} f^{2}}{\left(k^{2}+l^{2}\right)\left(\omega^{2}-l^{2} u_{\mathrm{A}}^{2}\right)}\right] \frac{\mathrm{d} \delta \hat{u}_{z}}{\mathrm{~d} z} .
$$

For a medium in which both $N$ and $u_{\mathrm{A}}$ are independent of $z$, Equation (5) reduces to

$$
\frac{\mathrm{d}^{2} \delta \hat{u}_{z}}{\mathrm{~d} z^{2}}+m^{2} \delta \hat{u}_{z}=0
$$

where

$$
m^{2}=\left(k^{2}+l^{2}\right)\left[\frac{N^{2}}{\left(\omega^{2}-l^{2} u_{\mathrm{A}}^{2}\right)}-1\right]\left[1-\left(\frac{\omega f}{\omega^{2}-l^{2} u_{\mathrm{A}}^{2}}\right)^{2}\right]^{-1}
$$

is the vertical component of the wavevector $\kappa=(k, l, m)$. In the ensuing discussion, we focus on waves with horizontal components of propagation in the $+\mathbf{e}_{y}$-direction, so that $\kappa=(0, l, m)$ with $l>0$. Equation (15) can be recast in the form of an equation for $\omega$, with solution

$$
\omega^{2}=l^{2} u_{\mathrm{A}}^{2}+\frac{1}{2}\left(\frac{N^{2} l^{2}+f^{2} m^{2}}{l^{2}+m^{2}}\right)+\frac{1}{2}\left[\left(\frac{N^{2} l^{2}+f^{2} m^{2}}{l^{2}+m^{2}}\right)^{2}+\frac{4 u_{\mathrm{A}}^{2} f^{2} l^{2} m^{2}}{l^{2}+m^{2}}\right]^{1 / 2},
$$

in agreement with the dispersion relation given by Hide (1969), for the assumed directionalities of $\mathbf{g}, \mathbf{B}_{0}$, and $\kappa$. Equation (16) contains as limiting cases a variety of wave modes, 


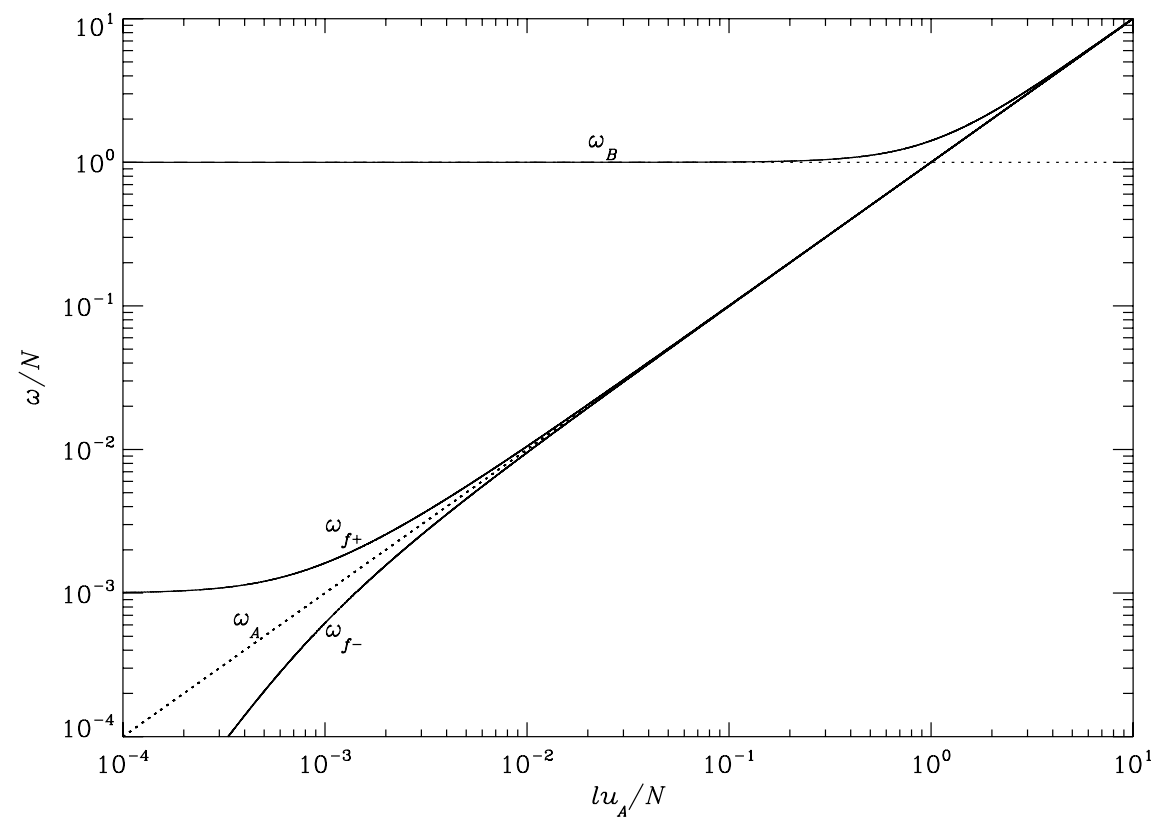

Figure 1 The regions in the $l-\omega$-plane for which gravity waves in a rotating, magnetized fluid can have a vertical component of propagation, as described in the text. The boundaries depicted correspond to rotation at a rate $f / N=10^{-3}$ ( $f$ is the Coriolis parameter and $N$ the buoyancy frequency) for waves with horizontal propagation in the $+\mathbf{e}_{y}$-direction. When $\mathbf{B}_{0}, f=0$, vertically propagating hydrodynamic gravity waves are possible for any combination of $l$ and $\omega$ for which $\omega / N<1$; that is, for the entire region below the horizontal dotted line. For MHD gravity waves in the absence of rotation $(f=0)$, vertical propagation is possible for waves within the region between the curve $\omega=\omega_{\mathrm{B}}$ and the diagonal dotted line for $\omega=\omega_{\mathrm{A}}$. When the effects of rotation are included $(f \neq 0)$, vertical propagation is possible within the regions bounded by the curves $\omega=\omega_{\mathrm{B}}, \omega_{f+}$ and $\omega=\omega_{\mathrm{A}}, \omega_{f-}$. Rotation becomes increasingly important at low frequencies and long horizontal wavelengths, causing the curves for $\omega=\omega_{f \pm}$ to diverge from each other.

including Alfvén waves $(N, f \rightarrow 0)$, hydrodynamic gravity waves $\left(u_{\mathrm{A}}, f \rightarrow 0\right)$, inertial waves $\left(N, u_{\mathrm{A}} \rightarrow 0\right)$, rotationally modified gravity waves $\left(u_{\mathrm{A}} \rightarrow 0 ;\right.$; e.g., Gill, 1982), MHD gravity waves ( $f \rightarrow 0$; e.g., Barnes, MacGregor, and Charbonneau, 1998), and MHD inertial waves $(N \rightarrow 0 ;$ e.g., Lehnert, 1954).

To gain insight into the propagation characteristics of MHD gravity waves in a rotating fluid, we examine the behavior of the vertical wavevector component $m$ as a function of $\omega$. From Equation (15), it is apparent that for fixed values of the quantities $N, u_{\mathrm{A}}, f$, and $l, m$ is real and vertical propagation is possible only for certain restricted ranges of $\omega$ values, as illustrated in Figure 1. Specifically, inspection of the expression for $m^{2}$ indicates that $m^{2}>0$ for

$$
\omega_{f+}<\omega<\omega_{\mathrm{B}} \text { and } \omega_{f-}<\omega<\omega_{\mathrm{A}},
$$

where

$$
\omega_{\mathrm{B}}=\left(N^{2}+l^{2} u_{\mathrm{A}}^{2}\right)^{1 / 2}, \quad \omega_{f \pm}= \pm \frac{1}{2} f+\frac{1}{2}\left(f^{2}+4 l^{2} u_{\mathrm{A}}^{2}\right)^{1 / 2}, \quad \omega_{\mathrm{A}}=l u_{\mathrm{A}} .
$$

For all other values of $\omega, m^{2}<0$ and the waves are evanescent, decaying exponentially in the $z$-direction. Figure 1 shows the regions of the $l-\omega$-plane within which the inequalities (17) 
are satisfied for $f / N=10^{-3}$. This value is representative of conditions near the base of the convection zone at a latitude of about $30^{\circ}$, since $\Omega \approx 2.8 \times 10^{-6} \mathrm{~s}^{-1}$ and $N \approx 2.5 \times 10^{-3} \mathrm{~s}^{-1}$, the latter quantity estimated from the standard solar model of Bahcall and Pinsonneault (1995).

From Equation (1) and the constraint $\nabla \cdot \delta \mathbf{B}=0$, the waves under consideration are transverse, $\kappa \cdot \delta \mathbf{u}=\kappa \cdot \delta \mathbf{B}=0$, so that both the velocity and magnetic fluctuations are contained in planes that are perpendicular to $\kappa$. If $\beta$ is the angle between a plane of constant phase and the vertical direction, then $\cos \beta=\left[l^{2} /\left(l^{2}+m^{2}\right)\right]^{1 / 2}$, where the variation of $m^{2} / l^{2}$ with frequency can be deduced from Equation (15). Hence, near the upper boundary in Figure 1 where $\omega \approx \omega_{\mathrm{B}}$, the waves behave like ordinary hydrodynamic gravity waves for $\left(l u_{\mathrm{A}} / N\right) \ll 1$, with $m^{2} / l^{2} \approx 0, \beta \approx 0$, and $\omega \approx N$, implying horizontal phase propagation and nearly vertical fluid motions. For $\left(l u_{\mathrm{A}} / N\right) \gg 1, \omega \approx l u_{\mathrm{A}}$, magnetic tension dominates the buoyant force, and the waves are like Alfvén waves that propagate in the direction of the background magnetic field. As the frequency is lowered at a fixed value of $l$, the fluid motions become increasingly horizontal $(\beta \approx \pi / 2)$, and buoyancy plays a smaller role in the wave dynamics. Near the line corresponding to $\omega=\omega_{f+}$ in Figure 1, the waves satisfy the approximate dispersion relation

$$
\omega^{2}-\left(\frac{m^{2}}{l^{2}+m^{2}}\right)^{1 / 2} f \omega-l^{2} u_{\mathrm{A}}^{2}=0,
$$

with $m^{2} / l^{2} \gg 1$. For $\left(f / l u_{\mathrm{A}}\right) \ll 1$, magnetic tension is again dominant and the waves are Alfvénic in character; when $\left(f / l u_{\mathrm{A}}\right)>1$, the Coriolis force controls the fluid motions and the disturbances are much like hydrodynamic inertial waves with $\omega \approx f$. At still lower frequencies, propagating waves (i.e., $m^{2}>0$ ) are possible within the region between the curves for $\omega=\omega_{\mathrm{A}}, \omega_{f-}$, wherein the approximate dispersion relation

$$
\omega^{2}+\left(\frac{m^{2}}{l^{2}+m^{2}}\right)^{1 / 2} f \omega-l^{2} u_{\mathrm{A}}^{2}=0
$$

holds. These waves have $\omega<l u_{\mathrm{A}}$, and for $\left(f / l u_{\mathrm{A}}\right)>1$ they are essentially the hydromagnetic inertial waves described by Lehnert (1954) (see also Acheson and Hide, 1973). As $\omega \rightarrow \omega_{f-}$, the fluid motions take place in planes that approach a horizontal orientation, with the waves becoming evanescent for $\omega<\omega_{f-}$.

Note that, like hydrodynamic gravity waves, the disturbances under consideration here have vertical phase and group speeds, $v_{\mathrm{p} z}$ and $v_{\mathrm{g} z}$, respectively, that are oppositely directed. In particular, by differentiating Equation (16), we obtain

$$
v_{\mathrm{g} z}=\frac{\partial \omega}{\partial m}=-\frac{m}{\omega}\left[\left(\omega^{2}-l^{2} u_{\mathrm{A}}^{2}\right)^{2}-(\omega f)^{2}\right]\left[N^{2} l^{2}+f^{2} m^{2}\left(\frac{\omega^{2}+l^{2} u_{\mathrm{A}}^{2}}{\omega^{2}-l^{2} u_{\mathrm{A}}^{2}}\right)\right]^{-1},
$$

from which it follows that for (say) $m>0, v_{\mathrm{p} z}=\omega m / \kappa^{2}>0$ while $v_{\mathrm{g} z}<0$ in both of the regions of vertical propagation delineated in Figure 1.

\section{Wave Reflection and Ducting}

We now use a simple model to investigate the reflection of vertically propagating MHD gravity waves in a layer containing a depth-dependent horizontal magnetic field. We consider small-amplitude, wave-like perturbations to a stationary, stratified medium in which 
the plane $z=0$ is a current sheet, separating the region (1) $z>0$ where $\mathbf{B}_{0}=B_{1} \mathbf{e}_{y}$ from the region (2) $z<0$ where $\mathbf{B}_{0}=B_{2} \mathbf{e}_{y}$, with $B_{1}$ and $B_{2}$ constant fields. We follow the approach described by Fan (2001) to ensure that the density $\rho_{0}$ of the unperturbed background varies continuously with $z$, despite the prescribed jump in the strength of the magnetic field at $z=0$. In particular, we express the background gas pressure and mass density in the form $p_{0}=p_{00}+p_{0 \mathrm{~B}}, \rho_{0}=\rho_{00}+\rho_{0 \mathrm{~B}}$, where $p_{00}$ and $\rho_{00}$ are the pressure and density in an unmagnetized isothermal medium, and $p_{0 \mathrm{~B}}$ and $\rho_{0 \mathrm{~B}}$ are the modifications to these quantities arising from the presence of the magnetic field $B_{0}(z)$. Magneto-hydrostatic equilibrium of the background medium then requires that $\mathrm{d} p_{00} / \mathrm{d} z=-g \rho_{00}$ and $\mathrm{d}\left[p_{0 \mathrm{~B}}+\left(B_{0}^{2} / 8 \pi\right)\right] / \mathrm{d} z=-g \rho_{0 \mathrm{~B}}$. By choosing $\rho_{0 \mathrm{~B}}=0$, it follows that $\rho_{0}$ is everywhere continuous and equal to the density of the unmagnetized medium, $\rho_{0}=\rho_{00}$. Integration of the second of the two equilibrium equations then yields $p_{0 \mathrm{~B}}=\left(B_{1}^{2}-B_{2}^{2}\right) / 8 \pi$, from which it can be seen that although a jump in the gas pressure exists at $z=0$, the total pressure, $p_{\text {tot }}=p_{0}+B_{0}^{2} / 8 \pi$, is continuous there. From the ideal gas law, the discontinuity in $p_{0}$ is associated with a jump $T_{0 \mathrm{~B}}=T_{00}\left(p_{0 \mathrm{~B}} / p_{00}\right)$ in the gas temperature at the current sheet location. The fractional changes in $p$ and $T$ are both $\approx \beta^{-1}$ and are small since $\beta=\left(8 \pi p_{00} / B_{0}^{2}\right) \approx 10^{6}$ for physical conditions like those at the base of the convection zone. The effects of compressibility have not been included in the analysis of Section 2; thus, the sound speed (i.e., $T_{0}$ ) does not appear in the dispersion relation (15), making that result applicable to the description of waves in either of the regions 1 and 2 delineated above.

We emphasize that the adopted background model is intended as an approximate representation of a neutrally buoyant, magnetic layer in the radiative region beneath the convection zone. We omit (among other things) treatment of the energy balance within the layer, assuming instead a piecewise-isothermal temperature distribution in the unperturbed medium that is consistent with the force balance that prevails there. However, this simplified model does allow us to isolate and explore magnetic effects on internal-wave propagation under solar-like conditions since, for $\rho_{0}$ continuous and $N$ assumed constant, Equation (15) indicates that a wave traveling from region 1 to region 2 will be affected solely by the discontinuity in $B_{0}$ at $z=0$. Acheson (1976) utilized a similar equilibrium model to investigate the over-reflection of hydromagnetic gravity waves in a medium that also contains a strong shear flow. An alternative treatment of the discontinuity in $p_{0}$ implied by the assumed $B_{0}(z)$, namely, a jump in $\rho_{0}$ with $T_{0}$ continuous at $z=0$, is discussed later in this section; in this case, wave propagation is affected by both the variations in $B_{0}$ and $\rho_{0}$.

We focus on a plane wave of frequency $\omega$ that originates in region 1 with $\kappa=(0, l, m)$, where $l$ and $m$ are both $>0$. Such a wave has its vertical component of propagation (i.e., $v_{\mathrm{g} z}$ ) in the direction of decreasing $z$ [see Equation (21), and the discussion thereof]; in traveling downward from region 1 into region 2, the wave will encounter a discontinuous change in the Alfvén speed, from $u_{\mathrm{A} 1}$ to $u_{\mathrm{A} 2}$, at $z=0$. We anticipate that, in general, both incident and reflected waves will be present in region 1 , while region 2 will contain a transmitted wave. The vertical velocity fluctuation arising from each of these components is a plane wave of the form $\delta u_{z}=\delta \hat{u}_{z} \exp [\mathrm{i}(l y-\omega t)]$, where $\delta \hat{u}_{z}$ satisfies Equation (5). For the conditions outlined in the preceding paragraph, it is clear that the relevant solutions to Equation (5) are

$$
\delta \hat{u}_{z}(z)=\delta \hat{u}_{\mathrm{I}} \exp \left(\mathrm{i} m_{1} z\right)+\delta \hat{u}_{\mathrm{R}} \exp \left(-\mathrm{i} m_{1} z\right)
$$

in region 1 , and

$$
\delta \hat{u}_{z}(z)=\delta \hat{u}_{\mathrm{T}} \exp \left(\mathrm{i} m_{2} z\right)
$$


in region 2, where $m_{1}\left(m_{2}\right)$ is the vertical wavevector component [see Equation (15)] in the upper (lower) region, and $\delta \hat{u}_{\mathrm{I}}, \delta \hat{u}_{\mathrm{R}}$, and $\delta \hat{u}_{\mathrm{T}}$ are the constant amplitudes of the incident, reflected, and transmitted waves at $z=0$.

The wave solutions in the upper and lower halves of the domain can be connected across the interface by applying physical conditions expressing the continuity of the vertical velocity and total pressure perturbations at $z=0$,

$$
\left[\delta \hat{u}_{z} \exp [\mathrm{i}(l y-\omega t)]\right]_{1}^{2}=0, \quad\left[\delta \hat{p}_{\text {tot }} \exp [\mathrm{i}(l y-\omega t)]\right]_{1}^{2}=0 .
$$

For $\delta \hat{p}_{\text {tot }}$ given by Equation (13) with $\rho_{0}$ assumed continuous at the interface, it follows directly from the imposition of these constraints that $\omega$ and $l$ are the same for the incident, reflected, and transmitted waves, and that

$$
\delta \hat{u}_{\mathrm{R}}=\left(\frac{1-q}{1+q}\right) \delta \hat{u}_{\mathrm{I}}, \quad \delta \hat{u}_{\mathrm{T}}=\left(\frac{2}{1+q}\right) \delta \hat{u}_{\mathrm{I}},
$$

where

$$
q=\frac{m_{2}}{m_{1}}\left(\frac{\omega^{2}-l^{2} u_{\mathrm{A} 2}^{2}}{\omega^{2}-l^{2} u_{\mathrm{A} 1}^{2}}\right)\left\{\frac{1-\left[\omega f /\left(\omega^{2}-l^{2} u_{\mathrm{A} 2}^{2}\right)\right]^{2}}{1-\left[\omega f /\left(\omega^{2}-l^{2} u_{\mathrm{A} 1}^{2}\right)\right]^{2}}\right\} .
$$

A quantity of particular relevance to this investigation is the reflection coefficient $[R]$ defined as

$$
R \equiv\left|\frac{\delta \hat{u}_{\mathrm{R}}}{\delta \hat{u}_{\mathrm{I}}}\right|=\left|\frac{1-q}{1+q}\right|,
$$

using the first of Equations (25).

We can develop a qualitative understanding of the reflection of MHD gravity waves by considering the ranges of $l$ and $\omega$ for which vertical propagation is possible in regions 1 and 2. The curves in Figure 2 delimit the portions of the $l-\omega$-plane in which $m_{1}$ (solid lines) and $m_{2}$ (dashed lines) are real, for the particular parameter values $u_{\mathrm{A} 2} / u_{\mathrm{A} 1}=5.0$, 0.2 and $f / N=10^{-3}$. In the following discussion, we focus on the particular case depicted in panel (a) for $\left(l u_{\mathrm{A} 1} / N\right)=10^{-2}$; analogous considerations can be applied to determine the propagation characteristics of waves corresponding to other values of $l$ and $u_{\mathrm{A} 2} / u_{\mathrm{A} 1}$. Proceeding from high to low frequencies along the vertical dotted line in the figure, the discussion of Section 2 indicates that propagating waves can exist in both regions 1 and 2 when $\omega_{f+2}<\omega<\omega_{\mathrm{B} 1} \approx \omega_{\mathrm{B} 2}$, where the frequencies defining this interval are given in Equations (18). In the range $\omega_{\mathrm{A} 2}<\omega<\omega_{f+2}$, waves can propagate in region 1 but are evanescent in region 2, while for $\omega_{f-2}<\omega<\omega_{\mathrm{A} 2}$, propagation is again possible in both regions. For $\omega<\omega_{f-2}$, wave propagation is precluded in region 2, while waves in region 1 are traveling for $\omega_{f+1}<\omega<\omega_{f-2}$ and $\omega_{f-1}<\omega<\omega_{\mathrm{A} 1}$ but evanescent for $\omega_{\mathrm{A} 1}<\omega<\omega_{f+1}$ and $\omega<\omega_{f-1}$.

The information obtained from Figure 2 concerning wave propagation on either side of the current sheet at $z=0$ can be used to infer something about the behavior of the reflection coefficient $R$ as a function of $l$ and $\omega$. For waves that can propagate in region 1 but are evanescent in region 2, $m_{2}^{2}<0$, and Equations (26) and (27) indicate that $q$ is imaginary and $R=1$. In this case, the discontinuity in $u_{\mathrm{A}}$ at the interface between regions 1 and 2 acts like a perfect reflector. For values of $l$ and $\omega$ such that vertical propagation is possible in both halves of the domain, $q$ is real, $R<1$, and the jump in $u_{\mathrm{A}}$ is partially reflecting. These deductions are verified by inspection of Figure 3, wherein we show the variation of $R$ with $\omega$ for $u_{\mathrm{A} 2} / u_{\mathrm{A} 1}=5.0$ and 0.2 , and $\left(l u_{\mathrm{A} 1} / N\right)=5 \times 10^{-4}, 10^{-3}, 10^{-2}, 5 \times 10^{-2}$; these 
Figure 2 MHD gravity-wave propagation in a medium with Alfvén speed $u_{\mathrm{A} 1}$ for $z>0$ and $u_{\mathrm{A} 2}$ for $z<0$. The curves are analogous to the boundaries identified in Figure 1, and delineate the portions of the $l-\omega$-plane where waves have $m^{2}>0$ in the upper (solid lines) or lower (dashed lines) half of the domain, as explained in the text. The results shown in the two panels were obtained for the parameter values $f / N=10^{-3}$ with $u_{\mathrm{A} 2} / u_{\mathrm{A} 1}=5.0$ (panel a) and 0.2 (panel b).

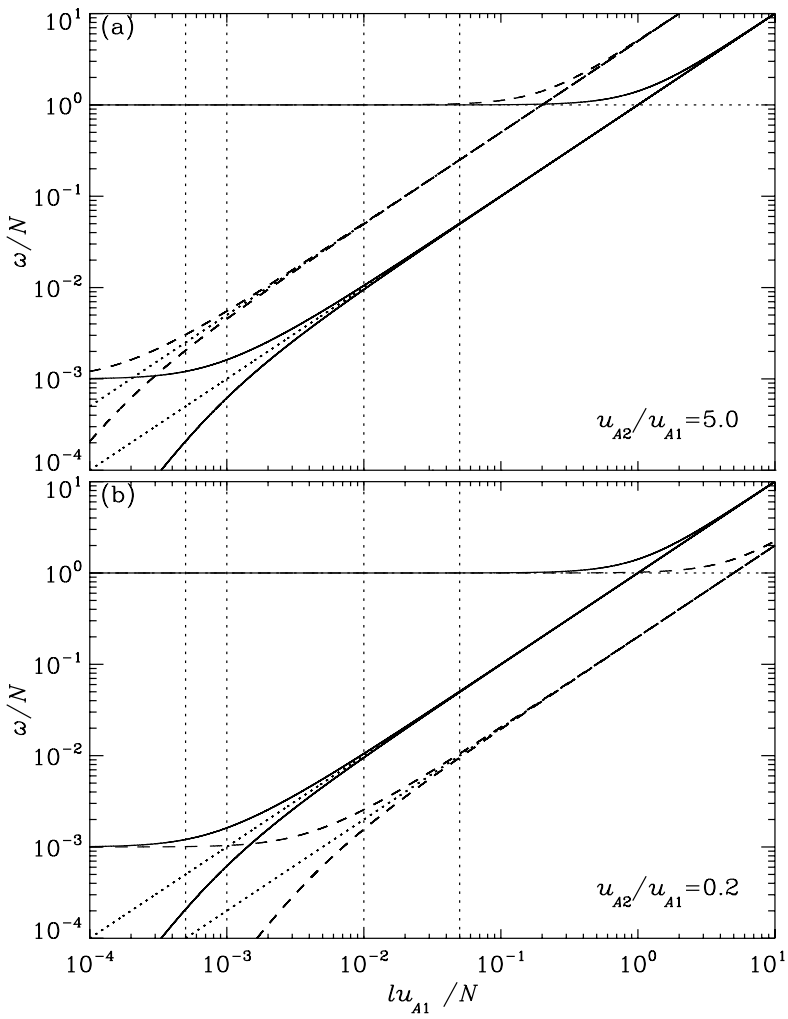

values of the horizontal wavevector component are indicated by the vertical dotted lines in Figure 2. For reference, note that if the mass density at the base of the convection zone is taken to be about $0.2 \mathrm{~g} \mathrm{~cm}^{-3}$, then $u_{\mathrm{A} 1} \approx 10^{4} \mathrm{~cm} \mathrm{~s}^{-1}$ for a field strength $B_{1}$ in the range $10-20 \mathrm{kG}$. With $N \approx 10^{-3},\left(l u_{\mathrm{A} 1} / N\right)=10^{-2}$ then implies that $l \approx 10^{-9} \mathrm{~cm}^{-1} \approx 2 \pi / H_{\mathrm{P}}$, where $H_{\mathrm{P}}\left(\approx 0.08 R_{\odot}\right)$ is the pressure scale height at the convection-zone bottom.

Referring to the discussion of the preceding paragraph and to Figure 2, it is readily seen from Figure 3 that within those frequency intervals for which $m_{1}^{2}>0$ and $m_{2}^{2}<0, R=1$. Likewise, at frequencies for which freely propagating waves are possible in both regions, $R<1$, with $R$ undefined at frequencies for which the waves are everywhere evanescent. As is apparent from the figure, as $\left(l u_{\mathrm{A} 1} / N\right)$ increases, the frequency intervals with $R=1$ shift to higher $\omega / N$, while the intervals with $R<1$ narrow. For downward propagation in a medium in which the strength of the horizontal field increases with depth, there are frequency intervals having $R=1$ for all values of $\left(l u_{\mathrm{A} 1} / N\right)$; in the case of a horizontal field strength that decreases with depth, perfect reflection occurs only for low frequency MHD inertial waves having $\left(l u_{\mathrm{A} 1} / N\right) \ll 1$. For frequencies at which the magnetic layer is partially reflecting, $R$ is a strongly varying function of $\omega$, with the interface becoming perfectly transmitting (i.e., $q=1$ and $R=0)$ for waves with $(\omega / N)^{2} \approx \frac{1}{2}\left\{1+\left(l u_{\mathrm{A} 1} / N\right)^{2}[1+\right.$ $\left.\left.\left(u_{\mathrm{A} 2} / u_{\mathrm{A} 1}\right)^{2}\right]\right\}$. Behavior analogous to the cases shown in Figure 3 is seen for other values of the ratio $u_{\mathrm{A} 2} / u_{\mathrm{A} 1}$, the only differences being in the extents of the frequency ranges for which the reflection is perfect or partial. For the special case in which $u_{\mathrm{A} 1}=0, u_{\mathrm{A} 2} \neq 0$, propagation in region 1 is possible for $\omega_{f+1}(=f)<\omega<\omega_{\mathrm{B}}(=N)$. Hence, for $\left(l u_{\mathrm{A} 1} / N\right)$ large enough that $\omega_{f-2}>f$, the variation of $R$ with $\omega$ for $\omega>\omega_{f-2}$ is as depicted for the 

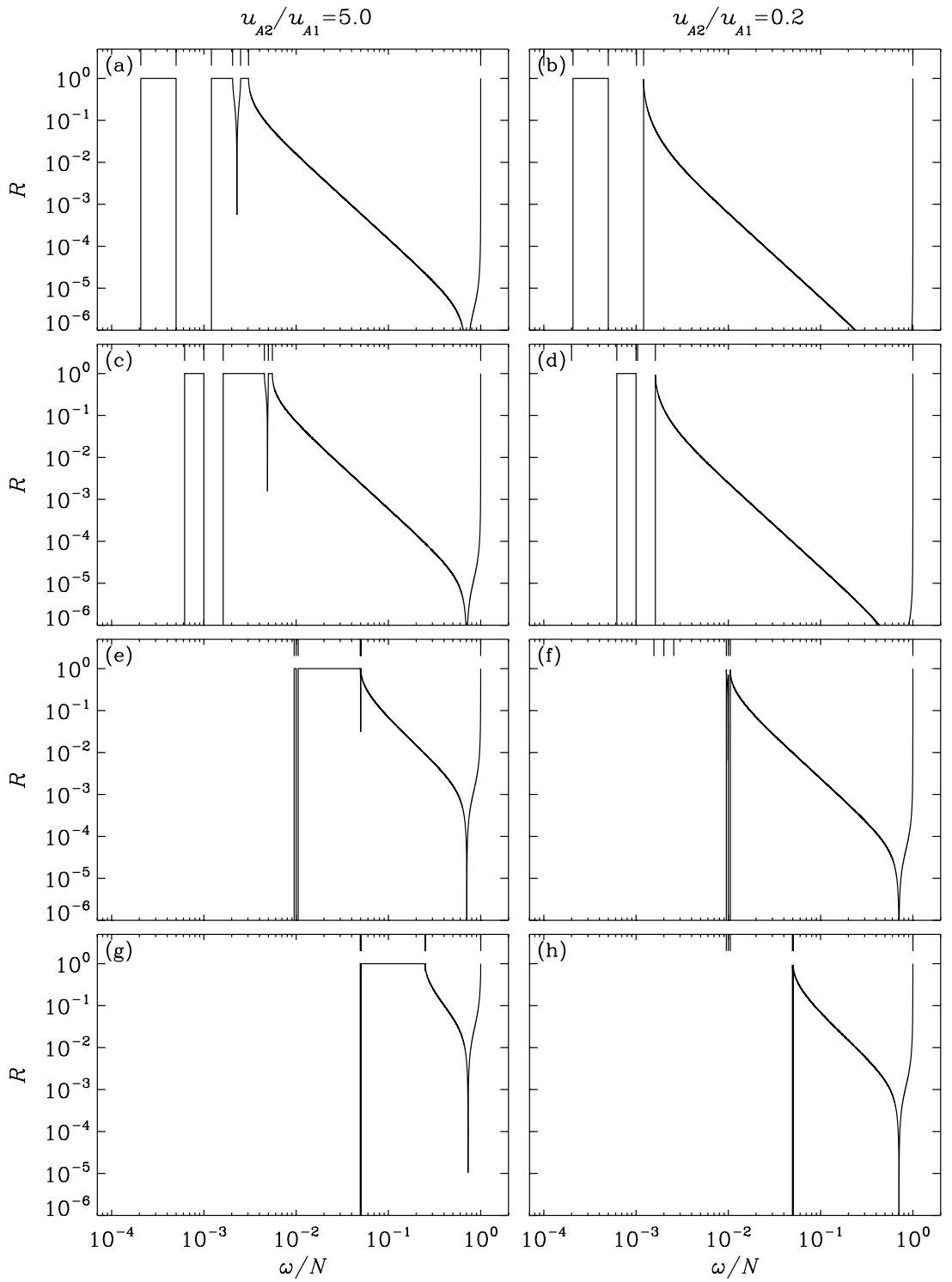

Figure 3 The reflection of MHD gravity waves in the composite medium of Figure 2. The left-hand (righthand) panels pertain to wave propagation in a medium with $u_{\mathrm{A} 2} / u_{\mathrm{A} 1}=5.0(0.2)$. The reflection coefficient $R$, defined as the ratio of the vertical-velocity amplitude of the reflected wave at $z=0$ to that of the wave incident there, is shown as a function of frequency for waves with $\left(l u_{\mathrm{A} 1} / N\right)=5 \times 10^{-4}$ (panels a and $\mathrm{b}$ ), $10^{-3}$ (panels $\mathrm{c}$ and $\mathrm{d}$ ), $10^{-2}$ (panels e and $\mathrm{f}$ ), and $5 \times 10^{-2}$ (panels $\mathrm{g}$ and $\mathrm{h}$ ); these values correspond to the vertical dotted lines in panels (a) and (b) of Figure 2. The long vertical ticks at the top of each panel indicate the frequencies $\omega_{f-}, \omega_{\mathrm{A}}, \omega_{f+}$, and $\omega_{\mathrm{B}}$ [see Equations (17) and (18) and the discussion thereof] that demarcate the frequency intervals within which wave propagation is possible for the given $l$ value in region $1\left(u_{\mathrm{A}}=u_{\mathrm{A} 1}\right)$ or region $2\left(u_{\mathrm{A}}=u_{\mathrm{A} 2}\right)$. 
cases with $u_{\mathrm{A} 2} / u_{\mathrm{A} 1}>1$ in Figure 3, with $R=1$ for $\omega_{f-2}>\omega>f$. For $\left(l u_{\mathrm{A} 1} / N\right)$ such that $\omega_{f-2}<f$, the lowest-frequency propagating waves in region 1 are partially reflected if $\omega_{f-2}<f<\omega_{\mathrm{A} 2}$ and perfectly reflected if $\omega_{\mathrm{A} 2}<f<\omega_{f+2}$.

The density and total pressure in the background equilibrium considered throughout this section are continuous across the current sheet at which the magnetic field, gas pressure, and temperature change discontinuously. For completeness, we note that the jump in gas pressure required to maintain equilibrium in the presence of the jump in $B_{0}$ could likewise have been provided by a jump in density with the temperature and total pressure continuous. For wave reflection in this case, with the density change across the sheet $\Delta \rho=\rho_{2}-\rho_{1}$ assumed small $\left(\Delta \rho / \rho_{0} \ll 1\right)$, the linearized continuity condition corresponding to the second of Equations (24) becomes (see, e.g., McKenzie, 1972; Delisi and Orlanski, 1975) $\left[\delta \hat{p}_{\text {tot }}-g \rho_{0} \xi\right]_{1}^{2}=0$, where $\xi=\mathrm{i} \delta \hat{u}_{z} / \omega$ is the amplitude of the wave-induced vertical displacement of the $z=0$ surface. Following a procedure analogous to that used in deriving the reflection coefficient (27), we find

$$
R=\left|\frac{1-q-\mathrm{i} Q}{1+q+\mathrm{i} Q}\right|
$$

where $q$ is still given by Equation (26) and

$$
Q=g \frac{\Delta \rho}{\rho_{0}} \frac{l^{2}}{m_{1}} \frac{\left(\omega^{2}-l^{2} u_{\mathrm{A} 1}^{2}\right)}{\left(\omega^{2}-l^{2} u_{\mathrm{A} 1}^{2}\right)^{2}-\omega^{2} f^{2}} .
$$

Note that for frequencies corresponding to waves that can propagate in region $1, m_{1}$ and $Q \propto\left(\Delta \rho / \rho_{0}\right) \propto \beta^{-1} \ll 1$ are both real quantities. For frequencies corresponding to waves that are evanescent in region 2, $m_{2}$ and $q$ are imaginary, and inspection of Equation (29) reveals that $R=1$, as was the case for reflection from a current sheet at which the density was continuous. For frequencies corresponding to waves that can propagate in both regions 1 and 2, $q$ is real and $>0$, and it is readily shown that $R<1$; as before, such waves are partially reflected. More specifically, in this case we find

$$
\left(\frac{1-q}{1+q}\right)^{2}<R^{2}=\frac{(1-q)^{2}+Q^{2}}{(1+q)^{2}+Q^{2}}<1,
$$

indicating that for frequencies at which partial reflection of the incident wave occurs, the reflection coefficient is enhanced by a small amount relative to the $R$ value that obtains for $\Delta \rho=0$ [cf. Equation (27)].

As described in Section 1, gravity waves are excited at and travel downward from the bottom of the convection zone; any upward propagation is prohibited by the superadiabatic stratification that prevails in the region above. Hence, reflection from a somewhat deeperlying magnetized layer could effectively confine the vertical propagation of waves having $R \approx 1$ to a thin slab-like region below the convection zone. To investigate the circumstances under which magnetic structure might contribute to the formation of such a duct or wave guide in the outermost layers of the radiative interior, we consider a configuration in which the strength of a $y$-directed magnetic field changes from $B_{1}$ to $B_{2}$ at depth $z=-d$ beneath the convection zone base $(z=0)$. Specifically, we identify the layers $-d \leq z \leq 0$ and $z \leq-d$ with the regions 1 and 2, respectively, of the previous discussion, and employ the solutions given in Equations (22) and (23) to describe the waves present in the upper and lower portions of the domain. As before, the continuity of the solutions at $z=-d$ is ensured 
by applying the conditions (24), and we utilize the requirement that $\delta \hat{u}_{z}(0)=\delta u_{0}$ to account for the excitation of waves at $z=0$.

Following the procedure outlined above, we find that the vertical component of the wave velocity is

$$
\delta \hat{u}_{z}=\delta u_{0} \frac{\exp \left[\mathrm{im} m_{1}(z+d)\right]+C_{\mathrm{R}} \exp \left[-\mathrm{i} m_{1}(z+d)\right]}{\exp \left(\mathrm{i} m_{1} d\right)+C_{\mathrm{R}} \exp \left(-\mathrm{i} m_{1} d\right)},
$$

in region 1 , and

$$
\delta \hat{u}_{z}=\delta u_{0} \frac{C_{\mathrm{T}} \exp \left[\mathrm{im} m_{2}(z+d)\right]}{\exp \left(\mathrm{i} m_{1} d\right)+C_{\mathrm{R}} \exp \left(-\mathrm{i} m_{1} d\right)},
$$

in region 2, where the coefficients $C_{\mathrm{R}}$ and $C_{\mathrm{T}}$ are

$$
C_{\mathrm{R}}=\frac{1-q}{1+q}, \quad C_{\mathrm{T}}=\frac{2}{1+q},
$$

with $q$ still given by Equation (26). The effectiveness of the layer $-d \leq z \leq 0$ (i.e., region 1 ) in functioning as a duct for gravity waves depends upon the magnitude of the reflection coefficient $[R]$ of the magnetic layer that serves as its lower boundary. For specificity, in the following we consider the case of wave propagation into a region of increasing horizontal field strength, as depicted in Figure 2a. From Figure 3, we can expect to trap little of the wave energy in the frequency band $\omega_{f-2}<\omega<\omega_{\mathrm{B} 1}$, since $R=1$ only in the narrow interval $\omega_{\mathrm{A} 2}<\omega<\omega_{f+2}$ with $R \ll 1$ everywhere else. However, the reflectivity of the bottom layer is considerably higher at lower frequencies, since all waves with $\omega<\omega_{f-2}$ that can propagate in region 1 are evanescent in region 2 and have $R=1$. We therefore expect that the duct that is formed when region 1 is sandwiched between the convection zone base on the upper side and region 2 below will suffer minimal leakage for propagating waves with $\omega_{f-1}<\omega<\omega_{f-2}$.

These expectations are confirmed by examining the amplitude of the total pressure perturbation $\delta \hat{p}_{\text {tot }}$ associated with MHD gravity waves in region 1 . Evaluating $\delta \hat{p}_{\text {tot }}$ using Equation (13) with the solution given in (31), we find

$$
\delta \hat{p}_{\mathrm{tot}}=-\frac{\rho_{0} \delta u_{0} m_{1}}{\omega l^{2}}\left(\omega^{2}-l^{2} u_{\mathrm{A} 1}^{2}\right)\left[1-\left(\frac{\omega f}{\omega^{2}-l^{2} u_{\mathrm{A} 1}^{2}}\right)^{2}\right]\left(P_{\mathrm{r}}+\mathrm{i} P_{\mathrm{i}}\right),
$$

where

$$
\begin{gathered}
P_{\mathrm{r}}=\frac{1}{D}\left(1-R^{2}\right) \cos \left(m_{1} z\right), \\
P_{\mathrm{i}}=\frac{1}{D}\left\{\left(1+R^{2}\right) \sin \left(m_{1} z\right)+2 C_{\mathrm{R}}^{\mathrm{r}} \sin \left[m_{1}(z+2 d)\right]-2 C_{\mathrm{R}}^{\mathrm{i}} \cos \left[m_{1}(z+2 d)\right]\right\},
\end{gathered}
$$

and

$$
D=1+R^{2}+2 C_{\mathrm{R}}^{\mathrm{r}} \cos \left(2 m_{1} d\right)+2 C_{\mathrm{R}}^{\mathrm{i}} \sin \left(2 m_{1} d\right) .
$$

In Equations (34)-(37), the reflection coefficient is defined as $R \equiv\left|C_{\mathrm{R}}\right|$, with $C_{\mathrm{R}}=$ $C_{\mathrm{R}}^{\mathrm{r}}+\mathrm{i} C_{\mathrm{R}}^{\mathrm{i}}$. In Figure 4 , we show the frequency dependence of the pressure fluctuation $\left|\delta \hat{p}_{\text {tot }}\right|$ at the tops (i.e., $z=0$ ) of ducts with vertical thicknesses $l d=10^{-1}$ and $l d=1$. For comparison with previous figures, the present results pertain to waves with $\left(l u_{\mathrm{A} 1} / N\right)=10^{-2}$ in a 

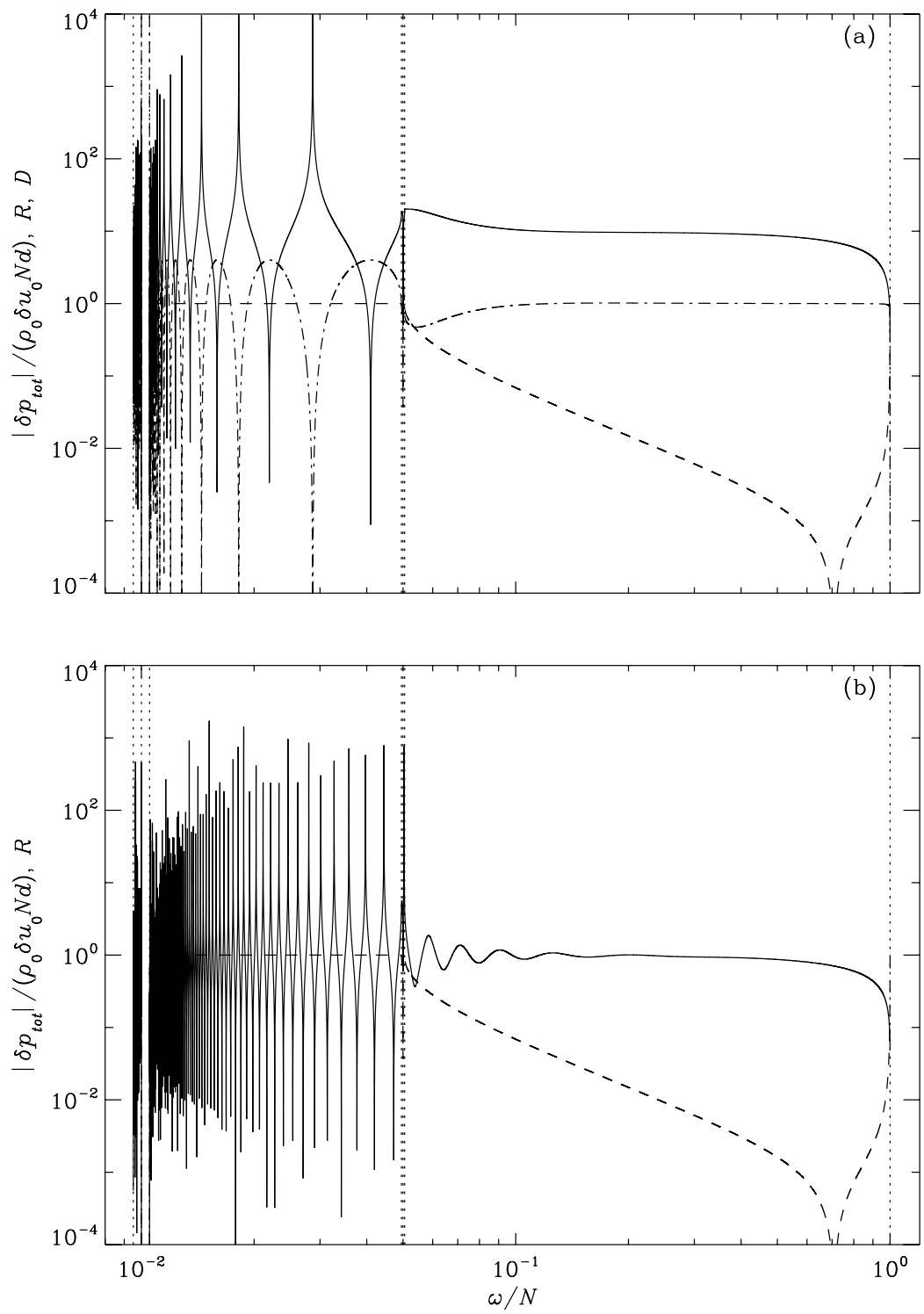

Figure 4 The amplitude of the total pressure perturbation [solid line; see Equation (13)] at $z=0$ for MHD gravity waves in a duct with vertical extent $d$, as discussed in Section 3 of the text. The results depicted were obtained for $\left(l u_{\mathrm{A} 1} / N\right)=10^{-2}, f / N=10^{-3}$, and $u_{\mathrm{A} 2} / u_{\mathrm{A} 1}=5.0$, with $l d=10^{-1}$ in panel a and $l d=1$ in panel $\mathrm{b}$. The dashed line in both panels represents the reflection coefficient $[R]$, while the dash-dotted line in panel (a) shows the value of the denominator $[D]$ given by Equation (37). The vertical dotted lines mark the frequencies given by Equations (18), evaluated in regions 1 and 2 (see also Figure 3).

medium with $f / N=10^{-3}$ and $u_{\mathrm{A} 2} / u_{\mathrm{A} 1}=5$.0. For the parameter values assumed throughout this discussion, the implied thickness of the ducting region when $l d=1$ is $d \approx 10^{9} \mathrm{~cm}$ $\approx 10^{-2} R_{\odot}$. 
As is apparent from the figure, at higher frequencies where $R \ll 1$, the waves are little affected by the change in magnetic conditions at the interface between regions 1 and 2 and propagate nearly freely. From the discussion of Section 2, for the adopted parameter values, these waves behave like hydrodynamic gravity waves, with $\left|\delta \hat{p}_{\text {tot }}\right| \approx \rho_{0} \delta u_{0} \omega m_{1} / l^{2} \approx$ $\rho_{0} \delta u_{0} N / l$. However, within the frequency intervals $\omega_{\mathrm{A} 2}<\omega<\omega_{f+2}, \omega_{f+1}<\omega<\omega_{f-2}$, and $\omega_{f-1}<\omega<\omega_{\mathrm{Al}}, R=1$, and the duct supports a sequence of wave modes, identifiable in the figure as significant enhancements in the value of $\left|\delta \hat{p}_{\text {tot }}\right|$ at discrete frequencies. The restoring force for these modes is a combination of the pressure gradient, and Coriolis and magnetic forces, the exact balance depending upon the magnitude of the quantity $\left(f / l u_{\mathrm{A} 1}\right)$ [see Equations (19) and (20), and the discussion thereof]. The vertical propagation of the waves is restricted to the layer $-d \leq z \leq 0$ by the structure of the duct, but they are able to propagate without attenuation in the horizontal direction. A similar behavior is seen in models for ducted hydrodynamic gravity waves in the Earth's atmosphere (see, e.g., Lindzen and Tung, 1976).

In the case under consideration, the peaks at which the total pressure perturbation is intensified are produced when waves in the layer that have opposite senses of vertical propagation constructively interfere. Inspection of panel a in Figure 4 reveals that the peaks coincide with the zeros of the denominator $D$, defined in Equation (37); these occur when

$$
2 m_{1} d-\theta=(2 n+1) \pi, \quad n=0,1,2, \ldots,
$$

where $\theta=\tan ^{-1}\left(C_{\mathrm{R}}^{\mathrm{i}} / C_{\mathrm{R}}^{\mathrm{r}}\right)$. Since $m_{1}$ increases with decreasing frequency [see Equation (15)], this relation yields a set of mode frequencies $\omega_{n}$ that decrease as $n$ gets larger. At fixed $l$, the horizontal phase speeds of these wave modes, $v_{\mathrm{p} y}=\left(\omega_{n} / l\right) /\left[1+(m / l)^{2}\right]$, become slower for increasing $n$. Condition (38) also establishes the vertical structure of the modes in the duct; for $(\theta / 4 \pi) \ll 1$, the wave with frequency $\omega_{n}$ has $(2 n+1) / 4$ vertical wavelengths within the duct width $d$. Taken together, these properties suggest that lowfrequency, high- $n$ modes are likely susceptible to radiative, viscous, or resistive dissipation. The peaks seen in Figure 4 occur for frequencies low enough that $R=1$; this means that the peak with the highest frequency has $n=3$ in the case $l d=10^{-1}$ and $n=13$ in the case $l d=1$. In this latter example, the peaks corresponding to lower values of $n$ are evident in the figure, albeit with reduced amplitudes resulting from the fact that the partially reflecting (i.e., $R<1$ ) lower boundary allows a fraction of the vertically propagating wave flux to escape from the duct.

It was pointed out in Section 2 that MHD internal waves are transverse, their associated fluid motions taking place in planes perpendicular to the direction of phase propagation. Due to the influence of the Coriolis force, $\delta \mathbf{u}$ does not maintain a fixed orientation within a given plane but instead rotates, giving the wave a helical structure (see, e.g., Moffatt, 1978). Waves of this kind may have some relevance to dynamo processes inside the Sun, since helical fluid motions are required in order to produce an $\alpha$-effect. The kinetic helicity of a single wave can be evaluated by computing the scalar product of the velocity fluctuation $\delta \mathbf{u}$ with the vorticity $\nabla \times \delta \mathbf{u}$. Using Equations (8) and (9) to determine $\delta \mathbf{u}$ for a freely propagating wave with $\delta u_{z}=\delta u_{0} \exp (\mathrm{i} \psi), \psi=\left(l y+m_{1} z-\omega t\right)$, we find

$$
\delta u_{x}=\delta u_{0}\left(\frac{\omega f}{\omega^{2}-l^{2} u_{\mathrm{A} 1}^{2}}\right) \frac{m_{1}}{l} \sin \psi, \quad \delta u_{y}=-\delta u_{0} \frac{m_{1}}{l} \cos \psi, \quad \delta u_{z}=\delta u_{0} \cos \psi,
$$

yielding

$$
\mathcal{H}_{\mathrm{K}}=\delta \mathbf{u} \cdot(\nabla \times \delta \mathbf{u})=-m_{1} \delta u_{0}^{2}\left(\frac{\omega f}{\omega^{2}-l^{2} u_{\mathrm{A} 1}^{2}}\right)\left(1+\frac{m_{1}^{2}}{l^{2}}\right)
$$




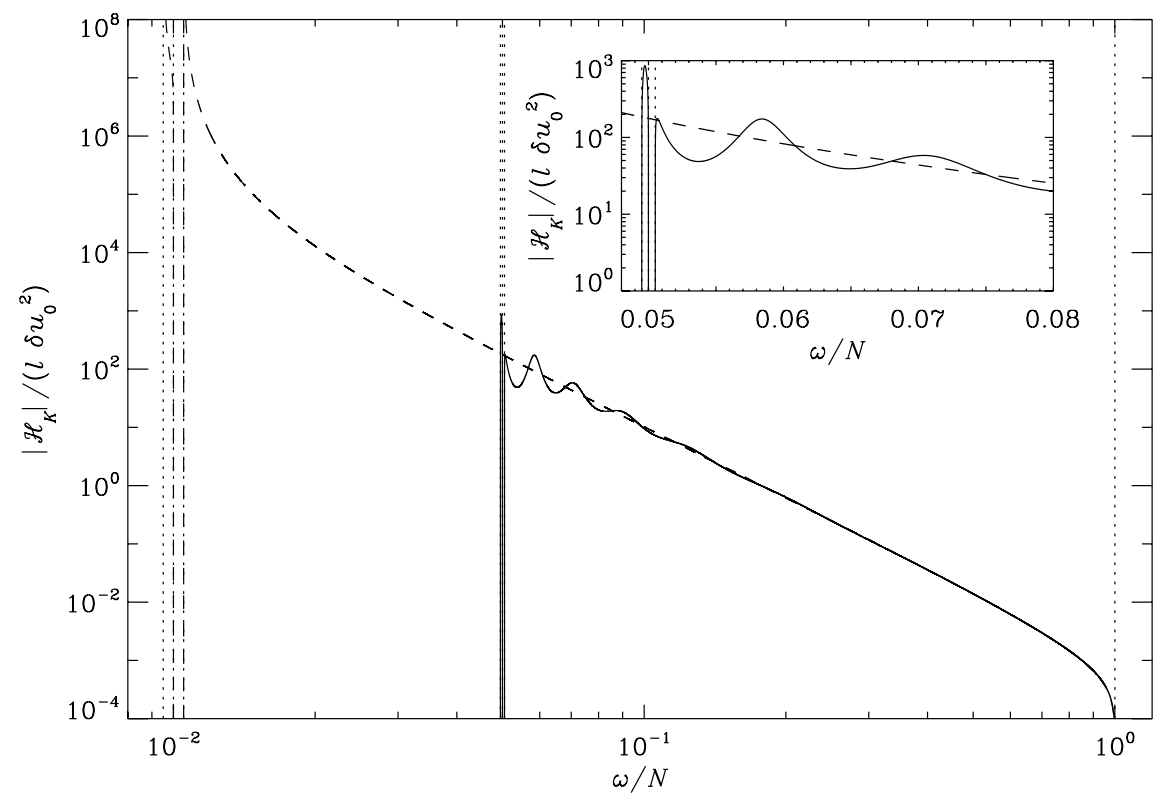

Figure 5 The absolute magnitude of the kinetic helicity of MHD gravity waves for $\left(l u_{\mathrm{A} 1} / N\right)=10^{-2}$ and $f / N=10^{-3}$. The dashed and solid lines represent the helicities associated with a wave that propagates without reflection [Equation (40)] and a ducted wave for the case $l d=1$ [Equation (41)], respectively. In the latter case, the lower boundary of the duct was taken to be the layer with $u_{\mathrm{A} 2} / u_{\mathrm{A} 1}=5.0$. The inset provides additional detail regarding the behavior of $\mathcal{H}_{\mathrm{K}}$ in the vicinity of the frequencies $\omega_{f+2}, \omega_{\mathrm{A} 2}, \omega_{f-2}$.

for the kinetic helicity. Similarly, for MHD gravity waves in a magnetically defined duct, we use Equation (31) for $\delta \hat{u}_{z}$ to obtain (after considerable manipulation)

$$
\mathcal{H}_{\mathrm{K}}=-m_{1} \delta u_{0}^{2}\left(\frac{\omega f}{\omega^{2}-l^{2} u_{\mathrm{A} 1}^{2}}\right)\left(1+\frac{m_{1}^{2}}{l^{2}}\right)\left(\frac{1-R^{2}}{D}\right),
$$

where $R$ is the reflection coefficient and $D$ is given by Equation (37).

In Figure 5, we show the absolute magnitudes of the kinetic helicities associated with both freely propagating and ducted waves, for $\left(l u_{\mathrm{A} 1} / N\right)=10^{-2}$ and $f / N=10^{-3}$, and for a duct with thickness $l d=1$; the results for $l d=10^{-1}$ are quite similar to those shown in the figure. From Equations (40) and (41), it is clear that when $f(=2 \Omega \cos \theta)>0$, the helicity of waves with $m_{1}>0$ that transport energy downward from the upper boundary is negative for $\omega>l u_{\mathrm{A} 1}$ and positive for $\omega<l u_{\mathrm{A} 1}$. The former of these frequency domains includes waves for which buoyancy contributes to the wave motion $\left(\omega_{f+1}<\omega<\omega_{\mathrm{B} 1}\right)$, while the latter corresponds to the hydromagnetic inertial waves $\left(\omega_{f-1}<\omega<\omega_{\mathrm{A} 1}\right)$. For decreasing $\omega$ in both frequency bands, $m / l$ becomes $\gg 1$, leading to wave-induced fluid motions that are increasingly horizontal and growing helicity. An example of this behavior is provided by gravity waves in the frequency interval $5 \times 10^{-2} \leq \omega / N \leq 5 \times 10^{-1}$, for which $\mathcal{H}_{\mathrm{K}} /\left(l \delta u_{0}^{2}\right) \approx$ $-(f / \omega)\left(m_{1} / l\right)^{3} \approx-f N^{3} / \omega^{4}$; at lower frequencies, for the hydromagnetic inertial waves, $\mathcal{H}_{\mathrm{K}} /\left(l \delta u_{0}^{2}\right) \approx(\kappa / l)^{3}$, where $\kappa=\left(l^{2}+m^{2}\right)^{1 / 2}$ is the total wavevector.

As is evident in the figure, the presence or absence of a reflecting interface has little impact on the wave helicity for $\omega_{f+2}<\omega<\omega_{\mathrm{B} 1}$ where $R<1$. Apart from the enhanced helicity of ducted waves with frequencies near the lower end of this interval, the results for 
both cases depicted in the figure are nearly indistinguishable. However, at lower frequencies, where waves are evanescent in region 2 and efficient reflection leads to the existence of the modes pictured in Figure 4, the helicity of the ducted waves vanishes. According to Equation (40), for waves with $\omega_{f+1}<\omega<\omega_{f-2}$, downward $\left(m_{1}>0\right)$ propagating waves have negative helicity and upward propagating waves $\left(m_{1}<0\right)$ have positive helicity. Hence, when $R=1$, the presence of equal fluxes of waves with opposite helicities in the duct yields zero net helicity, as indicated by Equation (41).

Because of their helical structure, it is of interest to ascertain whether these waves can contribute to the generation of a magnetic field through the production of an $\alpha$-effect. To do this, we follow Moffatt (1978) and consider the case in which wave propagation takes place within a weakly dissipative medium having magnetic diffusivity $\eta$. Using the fluctuating magnetic and velocity fields associated with a single freely propagating wave to calculate the mean electromotive force $\mathcal{E}=\langle\delta \mathbf{u} \times \delta \mathbf{B}\rangle=\alpha \mathbf{B}_{0}$, we find

$$
\alpha=\frac{\langle\delta \mathbf{u} \times \delta \mathbf{B}\rangle_{y}}{B_{0}}=-\left(\frac{\eta l^{2}}{\omega^{2}+\eta^{2} \kappa^{4}}\right) \mathcal{H}_{\mathrm{K}} .
$$

As indicated by Figure 5, this should also be a reasonable estimate of the $\alpha$-effect arising from waves that undergo partial reflection from the magnetic layer in region 2. Adopting $\eta=10^{9} \mathrm{~cm}^{2} \mathrm{~s}^{-1}$ for the diffusivity at the convection zone base and assuming $\mathcal{H}_{\mathrm{K}} /\left(l \delta u_{0}^{2}\right) \approx$ $10^{2}-10^{3}$ (Figure 5) with $l \approx 10^{-9} \mathrm{~cm}^{-1}$ and $\delta u_{0} \approx$ few $\times 10^{2} \mathrm{~cm} \mathrm{~s}^{-1}$ for $\omega / N \approx 5 \times 10^{-2}$, we obtain $\alpha \approx 10^{-2}-10^{-1} \mathrm{~cm} \mathrm{~s}^{-1}$.

\section{Summary and Discussion}

We have elucidated the physical properties of the internal waves that are likely to be present in the stable layers underlying the solar convection zone. We have used a simple, Cartesian model to conduct an exploratory examination of the reflection of vertically propagating waves in a region containing a horizontal (i.e., toroidal) magnetic field whose strength varies with depth; further investigation of these effects will require the use of a more realistic computational model (see, e.g., Rogers and MacGregor, 2010, 2011). For given values of $l$ and $\omega$, waves that travel downward from the base of the convection zone can undergo reflection with $R=1$ if they encounter a layer in which, because of the changing background magnetic conditions, they become evanescent. An effectively reflecting magnetized layer below the wave source region at the bottom of the convection zone can lead to the formation of a duct or wave guide, a structure that limits the vertical propagation of the perturbations and supports a set of horizontally propagating modes with enhanced amplitudes. In this regard, we note that inspection of the results for $R$ depicted in Figure 3 indicates that, for each value of the horizontal wavevector, there is a corresponding range of wave frequencies for which $R \approx 1$. If the energy flux of gravity waves excited by convective overshoot has the relatively flat distribution in frequency seen in the simulations of Kiraga et al. (2003) and Rogers and Glatzmaier (2005), then reflection could prevent a significant fraction of the emitted wave energy from reaching the deep solar interior. Such redirection and trapping of inward traveling waves would no doubt have implications for models in which the helioseismically inferred near-uniform rotation of the radiative interior is a long-term consequence of angular momentum redistribution by gravity waves (see, e.g., Charbonnel and Talon, 2005, and references therein). 
The internal waves studied here have helical structure, raising the possibility that such disturbances could contribute to the amplification of magnetic fields through dynamo action. Along these lines, Schmitt $(1984,1987)$ investigated a model in which unstable magnetostrophic waves, driven by magnetic buoyancy, provide the $\alpha$-effect for a dynamo located at the bottom of the convection zone. In the case we consider here, although the estimated $\alpha$-effect produced by a single freely propagating wave is small, the cumulative effect of a superposition of waves may be larger. The results of Figure 5 suggest that a further enhancement of the net helicity (and thus, of $\alpha$ ) of waves with $\omega \approx \omega_{f+2}$ might also be achieved through propagation in a leaky duct with a partially reflecting bottom. However, ducted modes for $R=1$ exhibit zero net helicity, a result of the cancellation of the positive and negative helicities of upward and downward traveling waves. In addition, mixing processes driven by these modes could affect the abundances of light elements in the outer layers of the radiative interior, although a quantitative assessment of these and related effects requires a more realistic representation of the structure of this region, together with treatment of such influences as the radiative damping of low frequency waves and irregularly shaped, non-horizontal reflecting surfaces (see, e.g., Phillips, 1963). A duct thickness $d \approx 10^{9} \mathrm{~cm}$ below the convection zone base represents an appreciable fraction of the distance over which mixing must occur in order to ensure destruction of lithium by nuclear processes.

Finally, our analysis did not directly account for the rotational shear flow that is the salient dynamical feature of the tachocline region. Note that in the presence of a mean background flow of the form $\mathbf{u}=u(z) \mathbf{e}_{y}$, the vertical components of the wavevector and group velocity become (see, e.g., Barnes, MacGregor, and Charbonneau, 1998)

$$
m^{2}=l^{2}\left[\frac{N^{2}}{(\omega-l u)^{2}-l^{2} u_{\mathrm{A}}^{2}}-1\right],
$$

and

$$
v_{\mathrm{g} z}=-\frac{m l^{2} N^{2}}{(\omega-l u) \kappa^{4}},
$$

respectively, where $\kappa=(0, l, m)$ and rotation has been neglected (i.e., $f=0)$, for simplicity. We consider the reflection of a wave that is incident on the interface between the region $z>0$ where $u_{\mathrm{A}}=u_{\mathrm{A} 1}$ and $u=u_{1}$, and the region $z<0$ where $u_{\mathrm{A}}=u_{\mathrm{A} 2}$ and $u=u_{2}$, adopting the configuration and nomenclature of Section 3. Utilizing the procedure given in Section 3 to derive the reflection coefficient, we again obtain $R=|(1-q) /(1+q)|$, but with

$$
q=\frac{m_{2}}{m_{1}}\left[\frac{\left(\omega-l u_{2}\right)^{2}-l^{2} u_{\mathrm{A} 2}^{2}}{\left(\omega-l u_{1}\right)^{2}-l^{2} u_{\mathrm{A} 1}^{2}}\right],
$$

instead of Equation (26).

For conditions such that waves are propagating in region 1 but evanescent in region 2, we anticipate that wave reflection should, apart from Doppler shifts arising from the advection of waves by the flow, qualitatively resemble the results obtained in Section 3 assuming $\mathbf{u}=0$. In particular, such a magnetized shear layer should support horizontally propagating, ducted modes of the kind investigated in Section 3, when we account for the effect of an overlying convective region on the vertical propagation of waves. Alternatively, note that if

$$
l^{2} u_{\mathrm{A} 1,2}^{2}<\left(\omega-l u_{1,2}\right)^{2}<N^{2}+l^{2} u_{\mathrm{A} 1,2}^{2},
$$

then by Equation (43) $m_{1}^{2}, m_{2}^{2}>0$ and vertical propagation is possible in both regions 1 and 2. For the case in which $u_{1}<\omega / l<u_{2}$, Equation (44) indicates that $v_{\mathrm{g} z}<0$ for the 
incident wave $\left(m_{1}>0\right)$ in region 1 but $v_{\mathrm{g} z}>0$ for the transmitted wave in region 2 , unless $m_{2}$ is chosen to be $<0$. However, with this choice, it follows from (46) that $q<0$ and $R>1$, so that the amplitude of the reflected wave exceeds that of the wave incident on the interface. In this case, the wave has undergone "over-reflection," with the amplitude of the reflected disturbance increased through interaction with the specified background shear flow (see Acheson, 1976 and references therein). From the preceding analysis, we conclude that this process requires $i$ ) the existence of a strong shear (in fact, the Richardson number $R i=[N /(\mathrm{d} u / \mathrm{d} z)]^{2}$ must be $<\frac{1}{4}$; see Acheson, 1976), ii) that the horizontal flow speed $[u]$ somewhere in the shear layer exceeds $\omega / l$, and iii) that vertical propagation must be possible on both sides of the interface $\left(m_{1}^{2}, m_{2}^{2}>0\right)$. Since the vertical shear within the tachocline region is thought to be characterized by $R i \gg \frac{1}{4}$ (Schatzman, Zahn, and Morel, 2000), it is unlikely that over-reflection of MHD gravity waves takes place. However, if the prevailing shear properties were conducive to the occurrence of over-reflection, then the magnetic shear layer would necessarily become unstable since, for waves in the appropriate frequency range, each successive reflection from the interface would increase the wave amplitude by a factor of $R$ ( $>1$; see also Acheson, 1976). Among the consequences of over-reflection of internal waves from the lower boundary of such a ducting region would be a nonzero net helicity for the modes in the presence of rotation (i.e., $f \neq 0$ ), since the differing amplitudes of the waves traveling in the $\pm \mathbf{e}_{z}$-directions imply that their respective helicities will be unequal in magnitude. Under these circumstances, it might be possible for the layer to function as a dynamo, amplifying the field through fluid motions whose energy source is the ambient shear flow.

Acknowledgements We are grateful to M. Dikpati, Y. Fan, P. Gilman, E.-J. Kim, and M. Miesch for many useful discussions concerning gravity waves in the solar radiative interior. We thank Hanli Liu for a critical reading of the manuscript, and an anonymous referee for comments that helped to clarify the model of Section 3. The National Center for Atmospheric Research is sponsored by the National Science Foundation.

\section{References}

Acheson, D.J.: 1976, J. Fluid Mech. 77, 433.

Acheson, D.J., Hide, R.: 1973, Rep. Prog. Phys. 36, 159.

Bahcall, J., Pinsonneault, M.: 1995, Rev. Mod. Phys. 67, 781.

Baldwin, M.P., Gray, L.J., Dunkerton, T.J., Hamilton, K., Haynes, P.H., Randel, W.J., et al.: 2001, Rev. Geophys. 39, 179.

Barnes, G., MacGregor, K.B., Charbonneau, P.: 1998, Astrophys. J. Lett. 498, L169.

Charbonneau, P., Tomczyk, S., Schou, J., Thompson, M.J.: 1998, Astrophys. J. 496, 1015.

Charbonneau, P., Christensen-Dalsgaard, J., Henning, R., Larsen, R.M., Schou, J., Thompson, M.J., Tomczyk, S.: 1999, Astrophys. J. 527, 445.

Charbonnel, C., Talon, S.: 2005, Science 309, 2189.

Delisi, D.P., Orlanski, I.: 1975, J. Fluid Mech. 69, 445.

Denissenkov, P.A., Pinsonneault, M., MacGregor, K.B.: 2008, Astrophys. J. 684, 757.

Fan, Y.: 2001, Astrophys. J. 546, 509.

Fisher, G.H., Fan, Y., Longcope, D.W., Linton, M.G., Pevtsov, A.A.: 2000, Solar Phys. 192, 119.

Fritts, D.C., Vadas, S.L., Andreassen, O.: 1998, Astron. Astrophys. 333, 343.

Garcia-Lopez, R.J., Spruit, H.C.: 1991, Astrophys. J. 377, 268.

Gill, A.E.: 1982, Atmosphere-Ocean Dynamics, Academic Press, New York.

Hide, R.: 1969, J. Fluid Mech. 39, 283.

Kim, E.-J., MacGregor, K.B.: 2001, Astrophys. J. Lett. 556, L117.

Kiraga, M., Jahn, K., Stepien, K., Zahn, J.-P.: 2003, Acta Astron. 53, 321.

Kumar, P., Talon, S., Zahn, J.-P.: 1999, Astrophys. J. 520, 859.

Lehnert, B.: 1954, Astrophys. J. 119, 647.

Lighthill, J.: 1978, Waves in Fluids, Cambridge University Press, Cambridge. 
Lindzen, R.S., Tung, K.-K.: 1976, Mon. Weather Rev. 104, 1602.

McKenzie, J.F.: 1972, J. Geophys. Res. 77, 2915.

Moffatt, H.K.: 1978, Magnetic Field Generation in Electrically Conducting Fluids, Cambridge University Press, Cambridge.

Phillips, O.M.: 1963, Phys. Fluids 6, 513.

Press, W.H.: 1981, Astrophys. J. 245, 286.

Press, W.H., Rybicki, G.B.: 1981, Astrophys. J. 248, 751.

Ringot, O.: 1998, Astron. Astrophys. 335, L89.

Rogers, T.M., Glatzmaier, G.A.: 2005a, Astrophys. J. 620, 432.

Rogers, T.M., Glatzmaier, G.A.: 2005b, Mon. Not. Roy. Astron. Soc. 364, 1135.

Rogers, T.M., MacGregor, K.B.: 2010, Mon. Not. Roy. Astron. Soc. 401, 191.

Rogers, T.M., MacGregor, K.B.: 2011, Mon. Not. Roy. Astron. Soc. 410, 946.

Rogers, T.M., Glatzmaier, G.A., Jones, C.A.: 2006, Astrophys. J. 653, 765.

Rogers, T.M., MacGregor, K.B., Glatzmaier, G.A.: 2008, Mon. Not. Roy. Astron. Soc. 387, 616.

Schatzman, E.: 1993, Astron. Astrophys. 271, L29.

Schatzman, E.: 1996, J. Fluid Mech. 322, 355.

Schatzman, E., Zahn, J.-P., Morel, P.: 2000, Astron. Astrophys. 364, 876.

Schmitt, D.: 1984, In: Guyenne, T.D., Hunt, J.J. (eds.) The Hydromagnetics of the Sun SP-220, ESA, Noordwijk, 223.

Schmitt, D.: 1987, Astron. Astrophys. 174, 281.

Schüssler, M.: 1996, In: Tsinganos, K.C. (ed.) Solar and Astrophysical Magnetohydrodynamic Flows, Reidel, Dordrecht, 17.

Spiegel, E.A., Veronis, G.: 1960, Astrophys. J. 131, 442.

Talon, S., Charbonnel, C.: 2005, Astron. Astrophys. 440, 981.

Talon, S., Kumar, P., Zahn, J.-P.: 2002, Astrophys. J. Lett. 574, L175.

Turner, J.S.: 1973, Buoyancy Effects in Fluids, Cambridge University Press, Cambridge. 